\title{
We Alain Didier-Weill, psychanalyste et dramaturge (16 juillet 1939-17 novembre 2018)
}

e 17 novembre 2018 Alain Didier-Weill est mort nous laissant une œuvre protéiforme dont nous n'avons pas terminé de tirer les conséquences théoriques, cliniques et éthiques.

Docteur en médecine et ancien interne des hôpitaux psychiatriques de la Seine, il fut l'analysant de Lacan qui l'invita à trois reprises à intervenir à l'occasion de son séminaire. Il fut tout d'abord psychanalyste membre de l'École freudienne de Paris (EFP); puis en 1983, suite à la dissolution de l'EFP, il cofonde le Mouvement du coût freudien, puis en 1991, l'Inter associatif européen de psychanalyse. En 2002, il fonde le Mouvement Insistance auquel est associé la revue qui porte le même nom. L'œuvre théorique conçue par Alain Didier-Weill est l'une des plus libres et des plus inventives de la fin du XXe et du début du XXI siècle. Elle a rencontré un important retentissement bien au-delà de nos frontières puisque son travail a été salué et très régulièrement commenté et traduit en Europe, mais également en Amérique du Nord et en Amérique du Sud. Alain Didier-Weill a, parallèlement à cette œuvre théorique, développé une œuvre dramaturgique qui est loin d'être anecdotique ${ }^{[2]}$ et qui s'articule de façon essentielle à ses élaborations théorico-cliniques. L'écriture théâtrale ne fut pas pour lui un simple passe-temps mais la mise en jeu et en scène des questions abordées à l'occasion de ses ouvrages théoriques. Le théâtre permettant alors de faire entendre encore et en corps ce que la théorie ne faisait parfois qu'esquisser. Voilà pourquoi il convient de lire Les trois temps de la loi avec Jimmy, Invocations avec Dionysos la naissance de l'acteur, Un mystère plus lointain que l'inconscient avec Vienne 1913... Enfin, ce n'est sans doute pas pour rien que le dernier

[1] Professeur de psychologie clinique et pathologique à l'université Nice Sophia Antipolis. Ses travaux sur la voix et la pulsion invocante l'ont conduit à discuter les thèses d'Alain Didier-Weill. C'est dans le cadre de ce compagnonnage qu'il a participé à la création et à l'aventure du mouvement Insistance à ses côtés.

[2] Le volume consacré à son œuvre théâtrale publié aux Éditions des crépuscules compte 745 pages! Sa première œuvre, Pol-fut mise en scène en 1975 dans le cadre du Festival d'Automne à Paris et reçut le prix U de la critique qui lui fut remis par Ionesco. Une de ses dernières pièces Freud-Einstein : 1933, a été filmée par Jean Queyrat dans une interprétation où se trouvent Michel Bouquet, Pierre Forest et Axelle du Rouret. Son théâtre a été régulièrement porté à la scène en France comme à l'étranger. 
ouvrage qu'il publia, Qu'est-ce que le surmoi?, se conclut par Pierre et Paul, La dernière nuit, une œuvre de théâtre, laissant ainsi, définitivement, le dernier mot au dramaturge.

Par ailleurs, Alain Didier-Weill a participé à la réalisation de deux films. Le premier, Quartier Lacan ${ }^{[3]}$, réunit les entretiens de figures de la psychanalyse qui furent en contact avec Lacan et parlent de lui (Jean Clavreul, Serge Leclaire, Wladimir Granoff, Moustafa Safouan, Charles Melman, Claude Dumézil, René Bailly, Maud Mannoni). Le second, The Caller, a été réalisé à New York par Richard Ledès sur un scénario d'Alain Didier-Weill. Ce film fut sélectionné pour le Festival du film de TriBeCa de New York en avril 2008 et y remporta le “ Made in NY " Narrative Award. L'œuvre théorique se dessine dans une saisissante clarté dès les trois interventions ${ }^{[4]}$ qu'il effectua à la demande de Lacan les 21 décembre 1976 et 8 février 1977 au cours du séminaire L'insu que sait de l'une-bésue s'aile à mourre et le 8 mai 1979 au cours du séminaire La topologie et le temps.

À cette occasion, il esquisse trois voies qui seront ensuite développées au cours des quarante années à venir. Ainsi l'intervention du 8 mai 1979 présente déjà ce qui constituera un des apports majeurs de sa pensée : une théorie du surmoi qui, tout en intégrant les perspectives freudiennes et lacaniennes, propose une lecture renouvelée de cette instance. Cette voie sera explorée et développée à l'occasion de ce que je considère comme étant la trilogie théorique qui structure toute l'ouvre d'Alain Didier-Weill : Les Trois temps de la loi ${ }^{[5]}$ (1995), Un mystère plus lointain que l'inconscient $^{[6]}(2010)$ et Qu'est-ce que le surmoi? Recherche clinique et théorique ${ }^{[7]}$ (2016). La théorie du surmoi qu'il propose est la suivante : sa manifestation se rencontrerait à l'occasion de trois temps ${ }^{[8]}$ (logiques et non chronologiques, même si cela n'est pas encore toujours très clair à l'occasion des Trois temps de la loi) qui seraient autant de possibilités pour le sujet désirant de ne pas insister. Alain Didier-Weill articule ces trois temps à trois injonctions allant de: "Pas un mot! (proféré par le surmoi archaïque qui renverrait à la position subjective de celui qui ne peut répondre: “Oui ! " au commandement symbolique lui proposant de " devenir parole, là où c'était »), à : “Vas-tu persévérer? »(interrogeant la possibilité pour le sujet de confirmer son insistance en persévérance), en passant par : “ N’insiste pas ! (porté par le deuxième surmoi qui interroge le moment troublant où le sujet choisit de

[3] Quartier Lacan, vidéo cassette réalisée en 1996 par Emil Weiss, diffusée par Le Seuil.

Ce film a donné lieu dans un second temps à un ouvrage où d'autres entretiens sont venus s'ajouter : Michèle Montrelay, Christian Simatos, René Tostain, René Major et Daniel Widlocher. Didier-Weill A., Weiss E., Gravas F. (2001), Quartier Lacan. Témoignages sur Jacques Lacan, Paris, Denoël.

[4] La transcription de ces trois interventions est consultable à l'adresse suivante: https://www.alaindidierweill.com/ archives/articles-et-textes-divers/trois-conf\%C3\%A9rences-donn\%C3\%A9es-au-s\%C3\%A9minaire-de-lacan/

[5] Didier-Weill A. (1995), Les trois temps de la loi. Le commandement sidérant, l'injonction du surmoi et l'injonction musicale, Paris, Seuil, Prix Edipe, 1997.

[6] Didier-Weill A. (2010), Un mystère plus lointain que l'inconscient, Paris, Aubier.

[7] Didier-Weill A. (2016), Qu'est-ce que le surmoi ? Recherche clinique et théorique, Toulouse, Érès.

[8] Didier-Weill A. (1995), opus cité, p. 29-111. 
recevoir la loi dans son versant symbolique et donc de commandement d'un devoir de devenir ou dans son versant surmoïque qui le conduira à désavouer en lui l'avenir de la parole).

Cette théorie du surmoi s'articule à une conception de la naissance du sujet qui implique, selon la formule de Lacan, " quelque chose qui va plus loin que l'inconscient ${ }^{[9]}$. La thèse que développe Alain Didier-Weill, après Lacan mais en en tirant de nouvelles conséquences théorico-cliniques ${ }^{[10]}$, est que la création par la parole a des limites qui implique qu'une partie du réel échappant au pouvoir de nomination restera pour toujours incréée menaçant de déborder la création. Le « à jamais irreprésentable " côtoiera pour toujours l'irreprésenté.

C'est à partir des premiers versets de la Genèse qu'Alain Didier-Weill illustre ce qui lui permettra d'introduire deux types d'appel complétant les ébauches lacaniennes concernant la théorie de la pulsion invocante. La théorie de la pulsion invocante (qu'Alain Didier-Weill choisira toujours d'écrire invoquante faisant lire le participe présent du verbe invoquer, mettant ainsi l'accent sur l'action du sujet et non sur ce qui qualifierait la pulsion) étant le second apport essentiel de son œuvre. C'est cette question que Lacan lui avait demandé de développer de façon quasi impromptue le 21 décembre 1976 dans une intervention qui s’intitulait : “Quand la musique nous entend : contribution à la question de la pulsion invoquante „ ${ }^{[11]}$.

Cette question est développée dans Les trois temps de la loi, mais se trouvera être précisée dans deux ouvrages : Invocations ${ }^{[12]}(1998)$ et Lila ou la lumière de Vermeer ${ }^{[3]}$ (2003). Alain Didier-Weill y démontre l'originalité de la place de la pulsion invocante dans la dynamique des pulsions et l'importance de sa prise en compte dans la conduite de la cure. Deux types d'appel présideraient à la naissance du sujet et auraient un rôle décisif quant à son développement. Dans le premier, l'appel serait soutenu par une voix silencieuse : un pur appel à advenir présidant à l'apparition même du réel. C'est celle que les Écritures présentifient dès le premier verset de la Genèse :

"Entête Elohîms créait les ciels et la terre " "14

À l'occasion du second appel une voix trouverait à s'exprimer dans une parole qui vise elle à mettre en forme ce réel advenu. C'est celle que nous croisons au troisième verset où la voix de Dieu se fait entendre et s'exprime dans une parole :

[9] «J'ai essayé d'introduire quelque chose qui va loin que l'inconscient ». Lacan, le 16 novembre 1976. Séminaire inédit, L'insu que sait de l'une-bévue s'aile à mourre.

[10] Ces conséquences sont développées dans les Mémoires de Satan où Alain Didier-Weill démontre comment le fait de mal faire le Bien peut entraîner le virulent désir de bien faire le Mal. Didier-Weill A. (2004) Mémoires de Satan, Paris, Flammarion.

[11] Cette intervention a été publiée dans Lila ou Lumière de Vermeer, prix CEdipe 2004.

Didier-Weill A. (2003) Lila ou la lumière de Vermeer. La psychanalyse à l'école des artistes, Paris, Denoël, p. 135-144.

[12] Didier-Weill A. (1998), Invocations. Dionysos, Moïse, saint Paul et Freud, Paris Calmann-Lévy.

[13] Didier Weill (2003), Lila ou la lumière de Vermeer, Opus cité.

[14] La Bible, Entête (La Genèse), traduite et commentée par André Chouraqui, Paris, Jean-Claude Lattès, 1992, p. 33. 
“Elohîms dit: "une lumière sera”."

Et c'est une lumière " ${ }^{[15]}$

La voix silencieuse et invocante sollicite le moment de surrection où le réel humain s'est trouvé enflammé par la rencontre avec l'adresse et la nécessité de devenir humain. La voix silencieuse est celle qui sollicite l'engagement du processus d'hominescence, la voix s'exprimant dans une parole le nomme et partant lui donne forme. Alain Didier-Weill nous conduit à repérer que la nomination ne suffit pas à elle seule à produire le processus du devenir humain. Il y faut également cet inespéré contenu dans la voix silencieuse qui ne prend pas appui sur une représentation mais sur une supposition.

Apparaît alors la troisième notion qui existait déjà à l'occasion des interventions au séminaire de Lacan et qui va nouer l'ensemble de l'œuvre : la question de la supposition.

Dès sa première intervention, en 1976, Alain Didier-Weill propose : “ Nous découvrons que la musique est une altérité qui aurait entendu en nous quelque chose que nous ne pouvions pas entendre par nous-même et qu'à ce titre, dans un premier temps logique, nous sommes moins l'auditeur de la musique qu'elle est notre auditrice. »Ce qu'Alain Didier-Weill indique ici est que la musique nous suppose, elle nous entend avant même que nous en soyons les auditeurs. Puis, il ajoute : " $\mathrm{Au}$ premier temps de la pulsion, où la musique cherche le sujet et trouve, en lui, ce qui, de lui, se prête à être entendu, succède avec le renversement ce que Freud repère comme l'apparition d'un nouveau sujet et d'un nouvel objet: alors que c'était la musique qui était en quête de ce qui lui manquait, quête d'un sujet récepteur, voici que c'est le sujet qui, avec le retournement pulsionnel, va se mettre en quête de ce qui lui manque auprès de la musique, qui devient ainsi sujet supposé savoir ce qu'est ce manque et où est ce manque. " En supposant le sujet, la musique lui permettrait d'acquérir un savoir sur le manque et sur le lieu où il se tient, son lieu-tenant. Ce premier texte nous introduit donc à ce que l'on pourrait appeler les enjeux de la supposition dont un des aspects me paraît essentiel : pour Alain Didier-Weill la supposition porte moins sur le savoir que sur le sujet. La non-tenue de la position éthique de la supposition impliquerait que l'on passerait d'un savoir supposé à un savoir imposé, comme cela se rencontre dans la position du maître ou celle de l’inquisiteur. Cette distinction sera précisée à l'occasion de son intervention en 1979 : “ Je dirais qu'un sujet supposé, c'est un sujet qui est éventuellement supposé pouvoir vous surprendre ; un sujet soupçonné, à l'encontre, c'est un sujet dont fondamentalement rien ne saurait surprendre venant de lui, puisqu'il y a, par rapport au sujet soupçonné, une prévention, une présomption plus exactement, et que rien de lui ne saurait surprendre : quoi qu'il dise, ça sera intégré quelque part et ça n’aura rien de surprenant. »

[15] Ibid., p. 41. 
Ainsi de 1976 à 1979 peut se lire la proposition suivante qui se déploierait en trois temps :

(1) Un sujet accueilli d'une certaine manière, supposé donc - et non su ou soupçonné -

(2) peut se découvrir, dans l'étonnement,

(3) pouvant acquérir un savoir inédit sur le manque qui le constitue.

Les trois temps de la supposition, pourrions-nous proposer. Trois temps qui conduiront Alain Didier-Weill à proposer en 1995 la très éclairante formule du sujet-supposé-savoir-qu'il-y-a-du-sujet. Le sujet-supposé-savoir-qu'il-y-a-du-sujet, s’il accepte d'être un sujet-supposé-savoir ne s'y réduit pas, autorisant une ouverture qui relance le procès de la subjectivation. Il permet un étonnement toujours renaissant car il offre au sujet la confrontation à une énigme : celle du désir et de sa cause. Cette réédition d'une rencontre non ratée est une des conséquences de la supposition. De fait, la supposition permet cette rencontre car elle se situe moins du côté de l'espoir que de l'inespéré. Inespéré que l'on peut définir comme l'existence d'une chose signifiante qui se révèle comme ce qui se trouve pouvoir rester, irrésistiblement, quand il ne reste plus rien de ce qui avait pu être espéré, comme ce qui est vectorisé par cette voix signifiante hors parole qui invite le sujet à advenir, là où le silence de l'attente conduit le moi à devoir répondre de sa possibilité d'existence.

Cette possible rencontre avec l'Autre, répétée et pourtant hors monotonie, Alain Didier-Weill l'a nommée " note bleue ». Note bleue qui conjugue la production d'un effet de révélation pour l'inconscient toujours identique et qui, pour autant, n'impose aucun des caractères de la compulsion de répétition. Là où la compulsion de répétition impose le retour du même, vécu lourdement, la supposition permet au sujet de faire l'expérience renouvelée d'une rencontre surprenante dans laquelle le sujet supposé se reconnaît entendu sans pour autant être pris au mot.

Position que Alain Didier-Weill incarna avec une élégance rare tout au long de sa vie. Tous ceux qui l'ont approché peuvent en témoigner. 